\title{
Einfluss von Betriebsgrößen auf die Schwingungsreduzierungswirkung im nasslaufenden Kupplungssystem: Empirische Modellbildung - Kennfelder und Skalierbarkeit
}

\author{
Arne Bischofberger ${ }^{1} \cdot$ Sascha Ott $^{1} \cdot$ Albert Albers $^{1}$ \\ Eingegangen: 14. Juni 2021 / Angenommen: 20. August 2021 / Online publiziert: 23. November 2021 \\ (c) Der/die Autor(en) 2021
}

\section{Zusammenfassung}

Nasslaufende Kupplungssysteme werden, mitunter durch die zunehmende Hybridisierung sowie die Automatisierung von Getrieben, auch in Zukunft in vielen Antriebsstrangtopologien, nicht nur im Bereich der Fahrzeugtechnik, vorhanden sein. Eine Erweiterung der Funktionen des Systems neben dessen Hauptfunktion, um z. B. eine Funktion zur Reduzierung von Schwingungen bietet sich daher umso mehr an. Durch die Verwendung der nasslaufenden Kupplung als Aktor zur Schwingungsreduzierung wird ermöglicht, konventionelle schwingungsmindernde Komponenten hinsichtlich Bauraum und Masse zu reduzieren. Das moderne nasslaufende Kupplungssystem bietet außerdem gegenüber gängigen Komponenten den bedeutenden Vorteil, eine Schwingungsreduzierung bedarfsgerecht einzusetzen und zu regeln.

Im Rahmen dieser Veröffentlichung werden Regressionsmodelle zur Beschreibung des Verhaltens von Bewertungsgrößen der Schwingungsreduzierungswirkung wie Amplitudenverhältnis und Winkelbeschleunigung in Abhängigkeit der Betriebsgrößen wie der Grundschlupfdrehzahl anhand eines tribologischen Systems empirisch ermittelt. Es werden Aussagen über die Skalierbarkeit sowie Gültigkeit der Modelle erarbeitet. Neben der Schwingungsreduzierungswirkung wird ergänzend auch die Verlustleistung im schlupfenden Kupplungssystem betrachtet.

Die erarbeiteten Modelle begünstigen das gezielte Einstellen und Auslegen zukünftiger Kupplungssysteme für die Funktionserweiterung Schwingungsreduzierung im bedarfsgerecht geregelten Dauerschlupfbetrieb unter Berücksichtigung des Zielkonflikts Komfort vs. Effizienz.

\section{Influence of operating parameters on the vibration reduction effect in the wet-running clutch system}

\begin{abstract}
Wet clutch systems will be present in many powertrain topologies also in the future, not only in automotive engineering, but also in many other areas, partly due to increasing hybridization and automation of transmissions. An extension of the system's functions in addition to its main function, e.g. using the system for vibration reduction, is therefore all the more suitable. The additional use of the wet-running clutch as an actuator for vibration reduction makes it possible to reduce conventional vibration-reducing components in terms of packaging space and mass. The modern wet-running clutch system also offers a major advantage over conventional components of being able to set and control vibration reduction on demand.

In this publication, regression models for describing the vibration reducing effect through rating values as a function of operating variables e.g. slip speed are determined empirically for one tribological system. Conclusions about scaling and the validation of the models are worked out. In addition to the vibration reduction effect, energy dissipation in the clutch system during slip operation is also considered. The obtained models support a targeted configuration of future clutch systems for an on demand vibration reduction in controlled slip operations with taking into account the objective of comfort vs. efficiency.
\end{abstract}

Arne Bischofberger

arne.bischofberger@kit.edu
IPEK - Institut für Produktentwicklung, Karlsruher Institut für Technologie (KIT), Kaiserstraße 10, 76131 Karlsruhe, Deutschland 


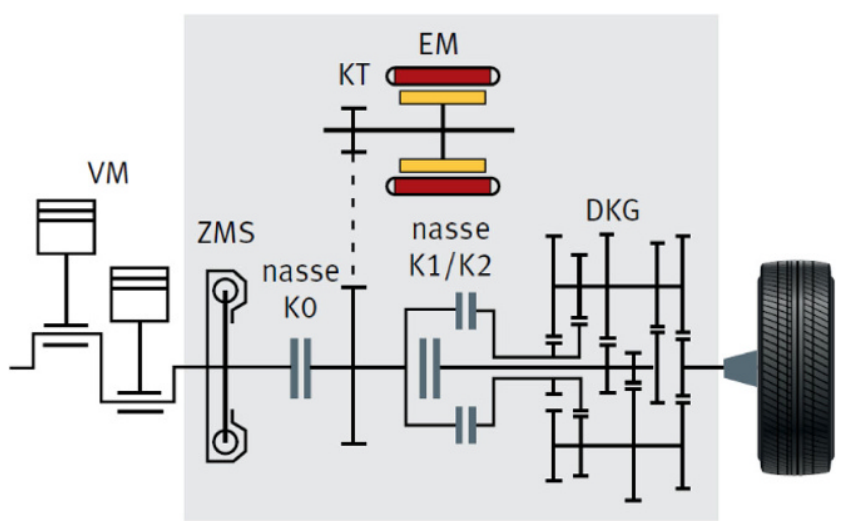

Abb. 1 Hybrider Antriebsstrang mit Dreifachnasskupplung [1]

\section{Einleitung und Stand der Forschung}

Neben herkömmlichen Komponenten zur Schwingungsreduzierung in Antriebssträngen, wie z. B. dem Zweimassenschwungrad (ZMS), bietet auch das moderne nasslaufende Kupplungssystem die Möglichkeit in Antriebssträngen Schwingungen reduzieren zu können. Verbaut als z. B. K0 im hybriden Antriebsstrang, vgl. Abb. 1, mit der Hauptfunktion den Leistungsfluss zwischen VKM und Restantriebsstrang herzustellen oder zu trennen, kann die Reduzierung von Schwingungen im gezielten Dauerschlupfbetrieb als Funktionserweiterung dank guter Steuer- und Regelbarkeit des modernen Kupplungssystems umgesetzt werden.

Die Erweiterung der Funktion der verbauten Kupplung bietet mitunter den Vorteil zukünftig Bauraum und Masse herkömmlicher Schwingungsreduzierungskomponenten reduzieren zu können. Entscheidend ist außerdem die gegenüber herkömmlichen Komponenten vorhandene Möglichkeit einer bedarfsgerechten Ansteuerung der Kupplung und damit eine gezielte Zuschaltung und Regelung der gewünschten Funktion.

Das Potenzial zur Schwingungsreduzierung durch das Kupplungssystem wird bereits u. a. durch [2-5] identifiziert und in verschiedenen Formen aufgezeigt. Albers [2] untersucht dabei ein elektronisches Kupplungsmanagement, in welchem die Kupplung als „schlupfgeregelter Filter“ betrachtet wird. Es wird eine Schlupfdrehzahl von $100 \mathrm{~min}^{-1}$ eingestellt und gezeigt, dass in diesem Betriebszustand nahezu keine Drehungleichförmigkeiten mehr über das Kupplungssystem übertragen werden. Auch in [6-8] wird das Potenzial zur Schwingungsreduzierung wieder aufgegriffen. In [7] wird die Isolationswirkung verschiedener Schlupfdrehzahlen untersucht. Auch hier wird eine nahezu vollständige Isolation bei einer Schlupfdrehzahl von ca. $100 \mathrm{~min}^{-1}$ ermittelt. Außerdem werden Isolationswirkungen eines ZMS mit und ohne Schlupfregelung gegenübergestellt. Es wird aufgezeigt, welche Schlupfdrehzahl bei einer kombinierten Anwendung mit konventionellem
ZMS notwendig ist, um am Getriebeeingang eine Zielamplitude von $5 \mathrm{~min}^{-1} \mathrm{zu}$ erreichen. Dabei wird ersichtlich, dass insbesondere im Bereich geringer Antriebsdrehzahlen $\left(<2000 \mathrm{~min}^{-1}\right)$ auch in Kombination mit einem ZMS verhältnismäßig hohe Schlupfdrehzahlen notwendig sind. Das ZMS weist in diesem Bereich eine schlechtere Isolationswirkung auf. In [6] wird die Reduzierung von Ungleichförmigkeiten für verschiedene Schlupfdrehzahlen an einer Einscheibentrockenreibkupplung untersucht. Bei einer Antriebsdrehzahl von $1000 \mathrm{~min}^{-1}$ wird auch hier eine praktisch vollständige Isolationswirkung erst bei hohen Schlupfdrehzahlen erreicht. Es werden daher Maßnahmen zur Schlupfbegrenzung vorgestellt. Dabei wird auch festgehalten, dass Kühlungsmaßnahmen notwendig sind, wenn die Reibleistung über einen längeren Zeitraum in etwa $0,5 \mathrm{~kW}$ übersteigt [6]. Eine nasslaufende Kupplung könnte sich hierfür und auch bereits bei geringeren permanenten Reibleistungen durch Dauerschlupfbetrieb zur günstigeren Energieabfuhr und Vermeidung von Verschleiß durch diesen neuartigen Betriebszustand besonders anbieten. Ergänzend zeigen Zhou et al. [9], dass auch eine „,mikroschlupfgeregelte" Kupplung (bei hier $\sim 20 \mathrm{~min}^{-1}$ Schlupfdrehzahl) die Drehungleichförmigkeiten im Antriebsstrang besonders in Bereichen geringer Antriebsdrehzahlen deutlich reduzieren kann. Hierbei wird die Winkelbeschleunigungsamplitude als Vergleichsgröße herangezogen.

Der Einfluss von Betriebsgrößen auf die Schwingungsreduzierungswirkung im nasslaufenden Kupplungssystem wird u.a. in [10] untersucht. Ein Einfluss kann, zu zuvor vorgestellten Untersuchungen in erster Linie durch die Schlupfdrehzahl, aber auch durch die Flächenpressung bzw. das Kupplungsmoment, sowie die Art der Anregung (Frequenz und Amplitude) festgestellt werden. Eine signifikant erhöhte Schwingungsreduzierungswirkung lässt sich dabei durch eine Erhöhung der Schlupfdrehzahl sowie eine Reduzierung der Flächenpressung bzw. des Kupplungsmoments feststellen. Ein Einfluss durch die Antriebsdrehzahl und den Kühlölvolumenstrom kann im Untersuchungsbereich hingegen nicht identifiziert werden. Um Aussagen zur Korrelation von Schwingungsreduzierung und Reibverhalten im Friktionskontakt treffen zu können, wird in [11, 12] außerdem der Einfluss der Betriebsgrößen auf das dynamische Reibungszahlverhalten untersucht. Eine mögliche Korrelation wird ergänzend in [10] untersucht. Nach [13] nimmt auch der Schmierstoff bzw. das Kühlöl maßgeblich Einfluss auf das Reibverhalten im Friktionskontakt. U. a. in [14] wird dazu auch der Einfluss des tribologischen Systems, mitunter der Kühlöls auf die Schwingungsreduzierungswirkung untersucht. Es kann auch hier ein signifikanter Einfluss identifiziert werden.

Um qualitative sowie quantitative Aussagen zum Einfluss der Betriebsgrößen treffen zu können, werden in [10, 14] lineare und erste nichtlineare Modelle für das Verhalten 
Abb. 2 Validierungsumgebung mit physischem Restsystem zur Untersuchung des Übertragungs- und Systemverhaltens nasslaufender Lamellenpakete im dynamischen Schlupfbetrieb unter Berücksichtigung der Systemwechselwirkungen, nach [16]

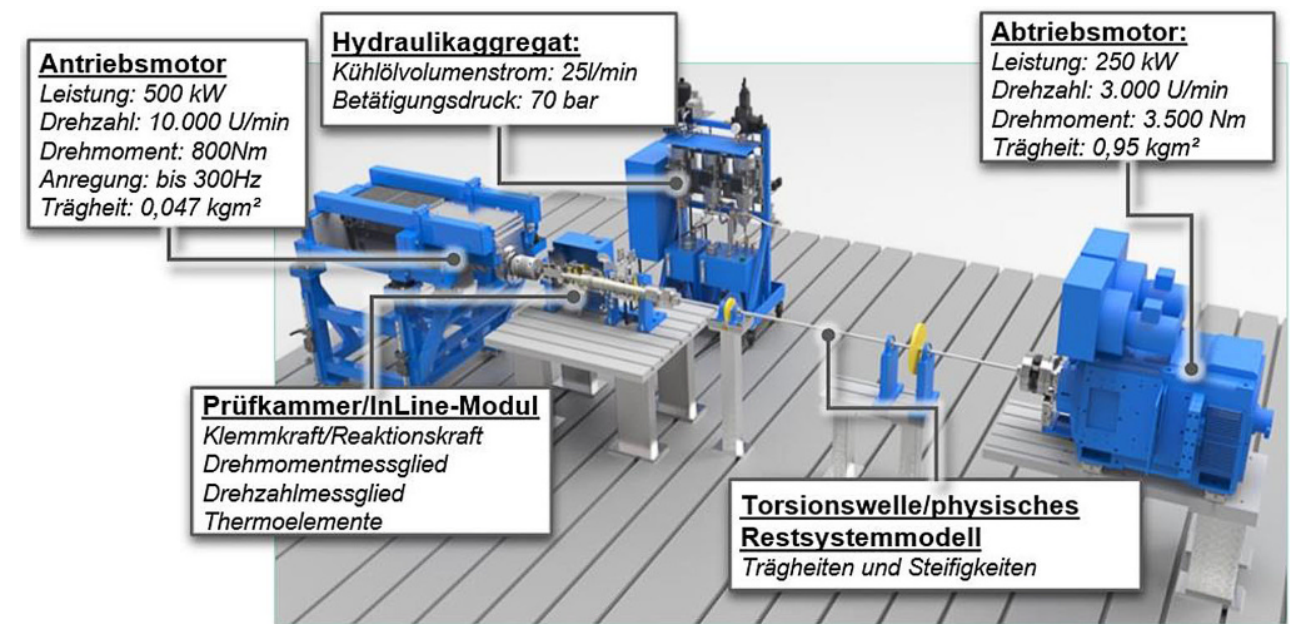

der Schwingungsreduzierungswirkung bei Veränderung der Betriebsgrößen wie der Schlupfdrehzahl und Flächenpressung bzw. Kupplungsmoment aufgestellt. Es werden hierbei verhältnismäßig geringe Bestimmtheitsmaße und damit eine geringe Modellgüte erreicht.

Um eine gewünschte Schwingungsreduzierungswirkung zu erreichen, ist eine gezielte Einstellung von BetriebsgröBen notwendig. Um diese hinreichend genau abschätzen und durchführen zu können, sind Modelle von höherer Modellgüte notwendig. Da die zugrundeliegenden Wirkmechanismen jedoch noch nicht tiefgehend ergründet sind und ohne Kenntnisse über das dynamische Reibverhalten im Tribosystem eine simulative Kennfeldermittlung nur stark vereinfacht möglich ist, ist ein eindeutiger, nichtlinearer physikalischer Zusammenhang nicht oder nur mit hohem Aufwand herstellbar. In dieser Veröffentlichung werden daher empirisch Modelle im dynamischen Dauerschlupfbetrieb erarbeitet. Die erarbeiteten Regressionsmodelle werden hinsichtlich deren Skalierbarkeit, bei z. B. Veränderung des Kupplungsmoments und deren Modellgrenzen bzw. Bereichen mit tendenziell unveränderten Wirkmechanismen validiert. Außerdem werden Kennfelder für die mögliche Anwendung in der Praxis erstellt und dabei auch Aussagen zur Verlustleistung im schlupfenden Kupplungssystem abgeleitet.

Die erarbeiteten Modelle und Kennfelder begünstigen es frühzeitig im Produktentstehungsprozess eine Abschätzung der vorgesehenen Maßnahmen zur Schwingungsreduzierung in Hinblick auf den gewünschten Zielkomfort treffen zu können. Außerdem vereinfachen die Modelle die spätere Erarbeitung genauer Einstellgrößen im gezielten Dauerschlupfbetrieb.

\section{Validierungsumgebung und Untersuchungsmethodik}

Durch simulative Untersuchungen wurde bereits ermittelt, dass das Reibverhalten im Friktionskontakt einen wesentlichen Einfluss auf das Übertragungsverhalten in diesem hat [15]. Um eine gezielte Auslegung und Einstellung des mechatronischen Aktors Kupplung sicherstellen zu können, sind detaillierte Kenntnisse über die Wirkzusammenhänge im Friktionskontakt notwendig [8]. Um die Wirkzusammenhänge und das Übertragungsverhalten im Friktionskontakt tiefgehend untersuchen zu können, wurde am IPEK Institut für Produktentwicklung am KIT eine Validierungsumgebung entwickelt und aufgebaut (Abb. 2).

Die Validierungsumgebung ermöglicht die Untersuchung des Übertragungsverhaltens im dynamischen Dauerschlupfbetrieb. Da ebenso triebstrangdynamische Wechselwirkungen mit dem Restantriebsstrang das Reib- und Übertragungsverhalten im Friktionskontakt beeinflussen [12], wird in der Validierungsumgebung ein Restsystemmodell integriert, welches eben diese Wechselwirkungen im Untersuchungsbereich hinreichend genau abbildet. Die Entwicklung und Validierung des Modells wird in [16, 17] detailliert vorgestellt. Eine ausführliche Vorstellung der Validierungsumgebung findet sich u. a. auch in [18].

Die Untersuchungen werden in der vorgestellten Validierungsumgebung mittels eines Lamellenpakets (vgl. Abb. 3) bestehend aus drei Stahl- sowie zwei Reiblamellen mit Papierreibelag und radialer Nutung (Baugröße $d_{I} / d_{A}=$ $158 / 188 \mathrm{~mm}$ ) durchgeführt. Außerdem zugehörig zum tribologischen System sind der Schmierstoff bzw. im Anwendungsfall nasslaufende Kupplung das Kühlöl sowie ein Beanspruchungskollektiv $[19,20]$. Auf dieses wird im späteren Verlauf genauer eingegangen. Als Kühlöl wird ein gängiges ATF (kinematische Viskosität bei $100^{\circ} \mathrm{C}: 4,2 \mathrm{mms} / \mathrm{s}$ ) verwendet. 
Abb. 3 Untersuchungsgegenstand Lamellenpaket, nach [10, 19]
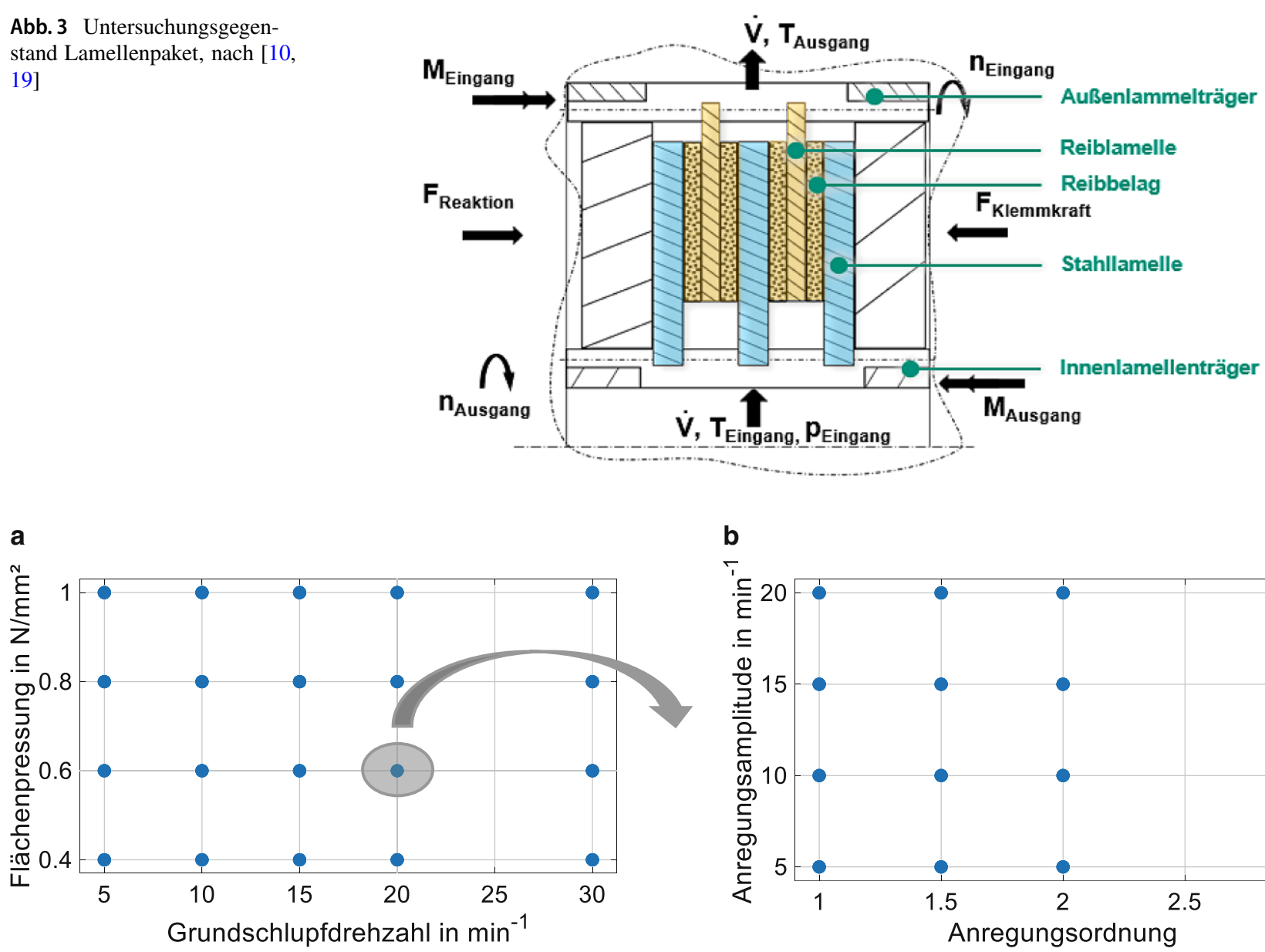

b

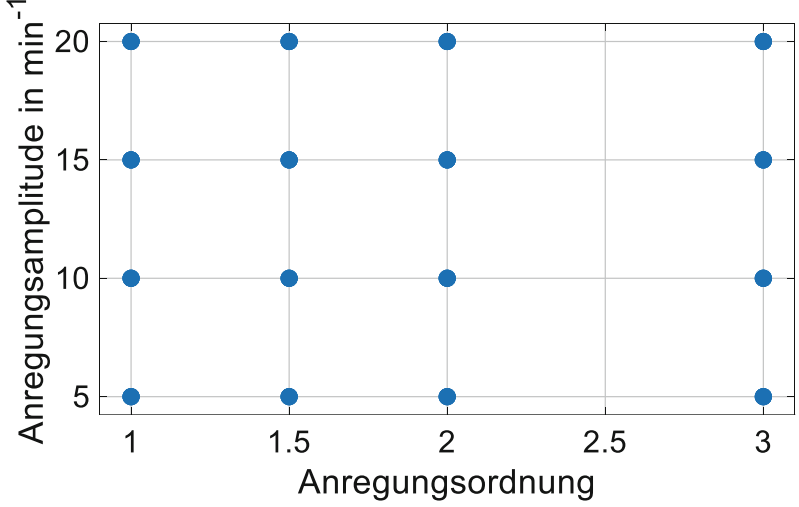

Abb. 4 a DoE: Parameterkombinationen für die Variation der Grundschlupfdrehzahl und Flächenpressung. b DoE: Parameterkombinationen für die Variation der Anregung

Die Untersuchungen werden im sogenannten dynamischen Dauerschlupfbetrieb durchgeführt. Es wird bei betätigter Kupplung (Flächenpressung) ein Dauerschlupf (Differenzdrehzahl zwischen An- und Abtrieb entsprechend Grundschlupfdrehzahl) eingestellt, eine sinusförmige Anregung entsprechend der Parameter aufgebracht und nach Einschwingen des Systems die eigentlichen Messgrößen erhoben. Im Detail wird das Vorgehen in [11, 12] beschrieben. Jede Parameterkombination wird viermal angefahren um Aussagen zur Wiederholbarkeit der Versuche treffen zu können. Zudem wird ein zweites Lamellenpaket mit Lamellen des gleichen Typs zur Bestätigung/Reproduzierbarkeit der Ergebnisse untersucht.

Um Aussagen zur Schwingungsreduzierungswirkung und Verbesserung dieser durch Veränderung von Betriebsgrößen treffen zu können, werden zwei Bewertungsgrößen herangezogen. Eine objektive Bewertung wird durch die Winkelbeschleunigung am Kupplungsausgang ermöglicht. Die Bewertungsgröße wird als Peak-to-Peak-Wert angegeben. Eine relative Bewertung wird durch das spezifische Amplitudenverhältnis ermöglicht. Dieses wird aus der Amplitude der Drehzahl an Kupplungsausgang sowie der Amplituden der Eingangsdrehzahl, bezogen auf den Frequenzbereich der Hauptanregenden, nach folgender Gleichung gebildet: $R_{A}=\widehat{n}_{\text {out }} / \widehat{n}_{i n}$. Für eine verbesserte Schwingungsreduzierungswirkung müssen beide Bewertungsgrößen schlussfolgernd minimiert werden. Die Bewertungsgrößen werden in [14] detailliert eingeführt.

Aus vorangehenden Untersuchungen werden für die Modellbildung relevante Parameterbereiche abgeleitet. Es wird ein Parameterraum für Variation der Betriebsgrößen Grundschlupfdrehzahl und Flächenpressung mit konstanter Anregungsordnung (2.Ordnung) und Drehzahlamplitude $\left(10 \mathrm{~min}^{-1}\right)$ um die jeweilige Antriebsdrehzahl aufgespannt. Die Betriebspunkte (Parameterkombinationen) sind in Abb. 4a dargestellt. Um Aussagen zur Skalierbarkeit bzgl. der Anregung treffen zu können wird zusätzlich ein weiterer Parameterraum für den in Abb. 4a markierten Betriebspunkt untersucht. Die gewählten Betriebspunkte zur Variation der Anregung sind in Abb. 4b dargestellt.

Der Kühlölvolumenstrom sowie die Antriebsdrehzahl werden aufgrund der Erkenntnisse aus [10] (kein signifikan- 
Abb. 5 Boxplot des spezifischen Amplitudenverhältnisses der jeweiligen vier Einzelversuche für jeden Betriebspunkt (Variation von Grundschlupfdrehzahl und Flächenpressung)

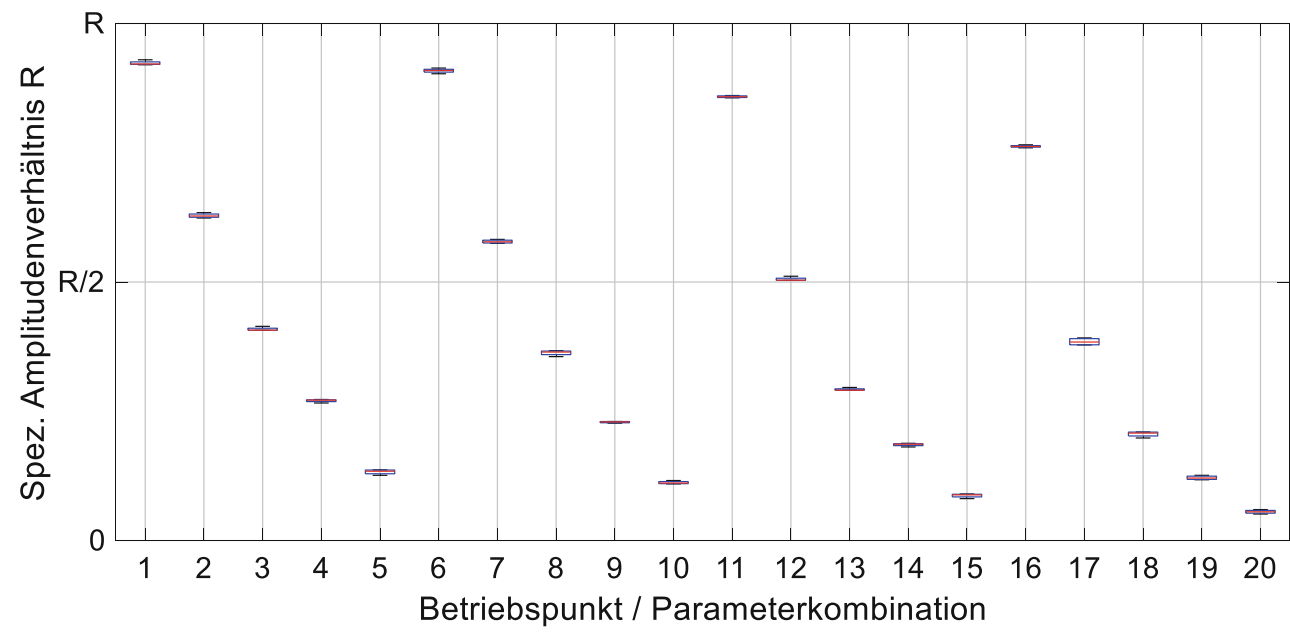

ter Einfluss im Untersuchungsbereich) in allen Versuchen konstant gehalten.

\section{Analyse der Messdaten: Wiederholbarkeit und Reproduzierbarkeit}

Um eine Wiederholbarkeit der Versuche überprüfen und sicherzustellen zu können, wird jede Parameterkombination im Versuch viermal angefahren. Die Wiederholbarkeit wird graphisch durch Auftragen der jeweiligen vier Messwerte eines Betriebspunkts anhand der Parameterkombinationen aus Abb. 4a verifiziert. Die entsprechenden Boxplots sind in Abb. 5 (spezifisches Amplitudenverhältnis) und Abb. 6 (Winkelbeschleunigung) aufgetragen.

Aus den Abbildungen wird ersichtlich, dass für die Winkelbeschleunigung tendenziell eine etwas höhere Streuung, bezogen auf den Untersuchungsbereich, vorliegt (vgl. z. B. Betriebspunkt 10). Die Streuung der Messwerte innerhalb eines Betriebspunkts liegt jedoch deutlich unter den Veränderungen der Bewertungsgrößen bei Veränderung der Be- triebsgrößen zwischen den einzelnen Parameterkombinationen. Es kann daher von einer Wiederholbarkeit der Ergebnisse ausgegangen werden.

Um zusätzlich eine Reproduzierbarkeit der Ergebnisse $\mathrm{zu}$ untersuchen, werden Bestätigungsversuche mit einem neuen Lamellenpaket gleicher Lamellentypen durchgeführt. Es wird dazu ein Wechsel des Lamellenpakets vorgenommen. Das Öl wird nicht gewechselt. Für jeden Betriebspunkt wird ein Boxplot aus den mittleren Messwerten der beiden Lamellenpakete ermittelt. Diese werden in nachfolgender Abb. 7 dargestellt.

Insbesondere im Bereich höherer Flächenpressungen (Betriebspunkte 1 bis 10) liegt eine, bezogen auf den Untersuchungsbereich, sichtbare Abweichung vor. Eine Erklärung hierfür kann in geringen Abweichungen der anliegenden Ist-Netto-Flächenpressung zwischen den beiden Lamellenpaketen liegen. Dies kann zukünftig mit einer auf das Kupplungsmoment normierten Bewertungsgröße verifiziert werden. Die Abweichungen können mitunter auch aus Ölalterungseffekten resultieren, da das Öl als Bestandteil des tribologischen Systems ebenso Einfluss auf
Abb. 6 Boxplot der Winkelbeschleunigung der jeweiligen vier Einzelversuche für jeden Betriebspunkt (Variation von Grundschlupfdrehzahl und Flächenpressung)

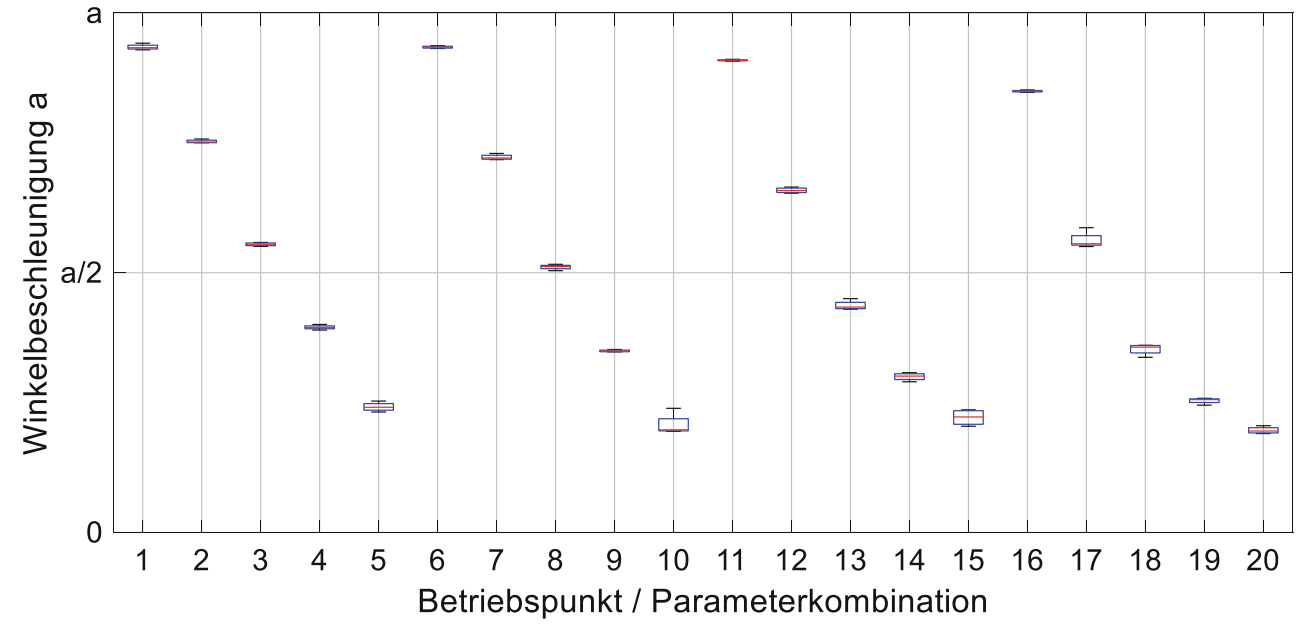


Abb. 7 Boxplot des spezifischen Amplitudenverhältnisses für zwei baugleiche Lamellenpakete

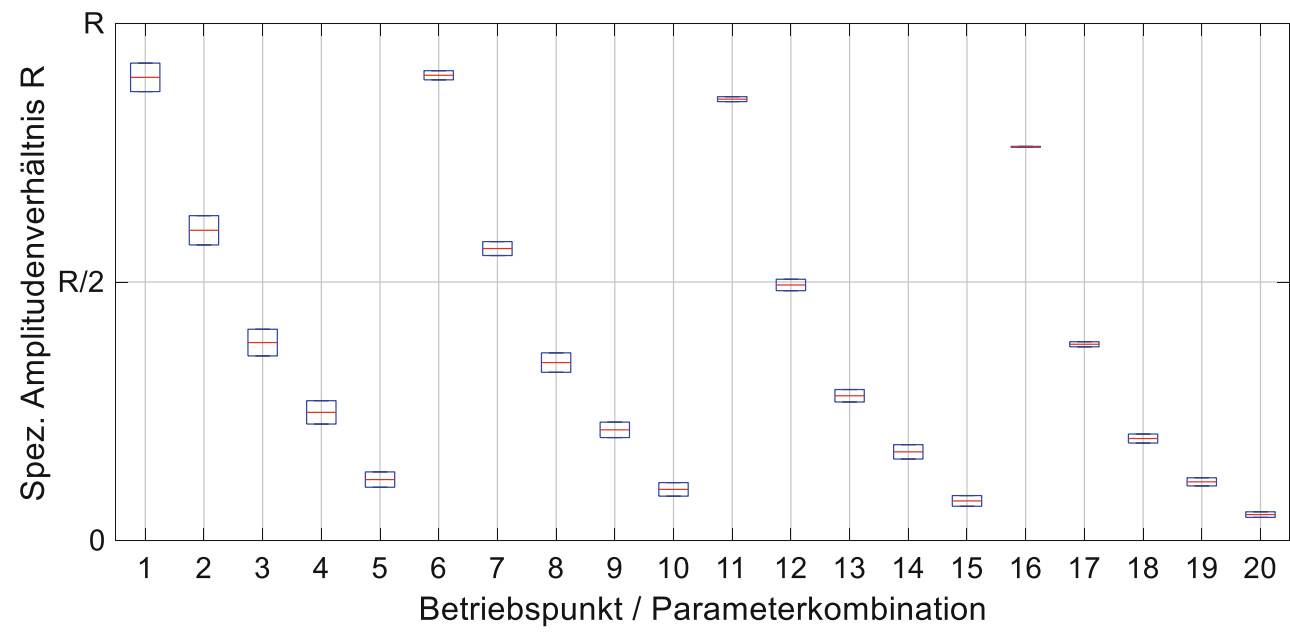

die Bewertungsgrößen nimmt und im Rahmen der Untersuchungen nicht ausgetauscht wird. Im Rahmen der Untersuchungen kann aufgrund der geringen Abweichungen dennoch von einer Reproduzierbarkeit der Ergebnisse für ein baugleiches Tribosystem ausgegangen werden.

\section{Einfluss von Betriebsgrößen: Empirische Modellbildung}

In folgendem Kapitel werden anhand der zuvor ermittelten Messdaten empirisch Regressionsmodelle für den quantitativen Einfluss der untersuchten Betriebsgrößen auf die Schwingungsreduzierungswirkung im untersuchten tribologischen System gebildet. Die Modelle sollen die gezielte Einstellung von Betriebsgrößen im nasslaufenden Kupplungssystem in Hinblick auf die Funktionserweiterung „Schwingungsreduzierung“ begünstigen.
Für die Modellbildung werden die empirisch ermittelten Daten von jeweils drei Stützpunkten, welche im Versuch jeweils viermal angefahren werden (vgl. Wiederholbarkeit Kap. 3), herangezogen. Durch die Verwendung dreier Stützpunkte können gegenüber der Verwendung von nur zwei Stützpunkten auch nichtlineare Ansatzfunktionen berücksichtigt werden. Die vierte und teilweise fünfte Stützstelle dient der Validierung des Modells in interpolierten, bei fünf Datenpunkten auch extrapolierten, Bereichen. Die Modellbildung wird grundlegend aus vier Teilschritten aufgebaut:

1. Auswahl geeigneter Ansatzfunktionen,

2. Parametrierung: Berechnung der Regressionsmodelle mittels experimentell ermittelter Stützpunkte,

3. Validierung in interpolierten (und extrapolierten) Bereichen anhand experimentell ermittelter Daten,

4. Ableitung von Aussagen zur Gültigkeit der Modelle. a
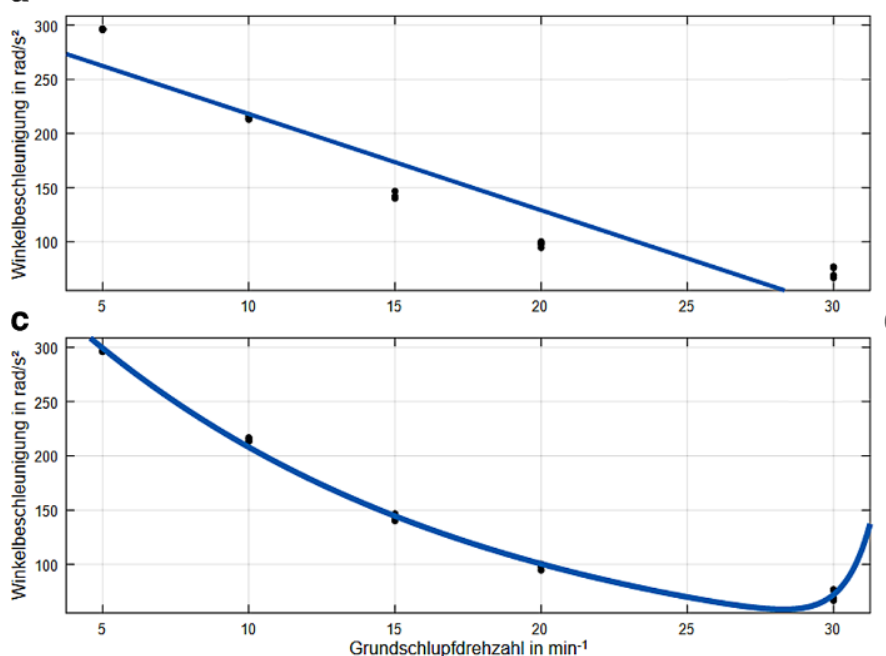

b
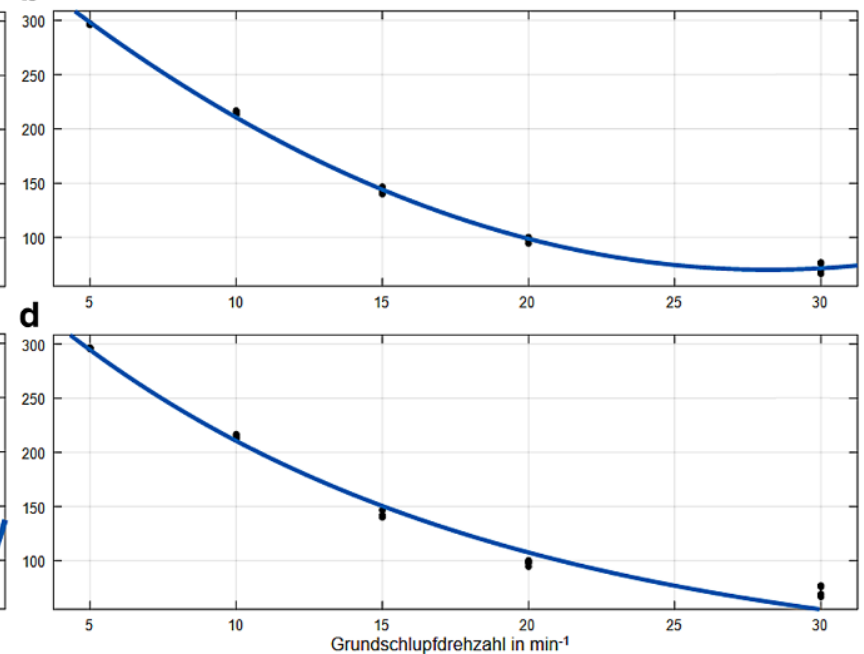

Abb. 8 Verlauf möglicher Ansatzfunktionen: a linear, b polynomial 2. Grades, c exponentiell (zwei e-Terme), d exponentiell (ein e-Term) 
Abb. 9 Angenäherter Verlauf des spez. Amplitudenverhältnisses über der Grundschlupfdrehzahl, Regressionsmodell aus 3 Stützstellen, $\mathrm{R}^{2}$ : 99,7\%; (Die Kurven mit 5 Stützstellen sind zur besseren Sichtbarkeit ausgeblendet, diese werden im Kapitel Validierung eingeblendet und mitbetrachtet.)

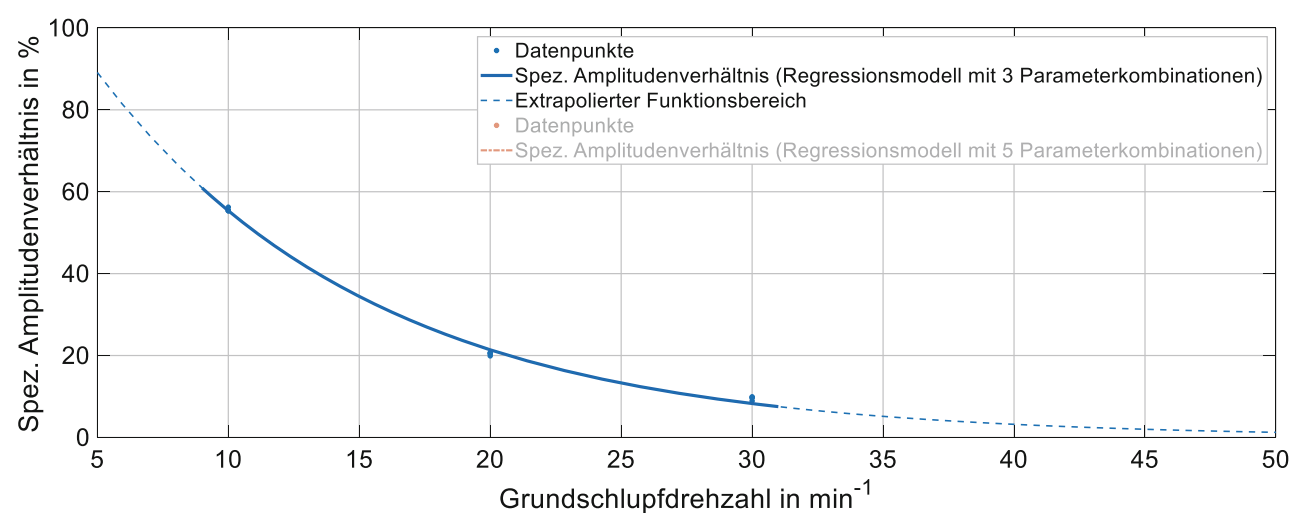

$\mathrm{Zu}$ Beginn werden geeignete Ansatzfunktionen ausgewählt. Anschließend werden die Modelle parametriert. Mittels der ausgewählten Ansatzfunktionen werden dazu aus den Versuchsdaten die Regressionsmodelle berechnet. Im nächsten Schritt wird eine Validierung der Modelle mittels zusätzlicher experimentell ermittelter Datenpunkte durchgeführt. Im abschließenden Schritt werden Modellgrenzen untersucht und eine Aussage zur Skalierbarkeit sowie Gültigkeit der Modelle abgeleitet.

Die Auswahl einer geeigneten Ansatzfunktion wird hier exemplarisch anhand des Verlaufs einer Bewertungsgröße für die Schwingungsreduzierung über der Grundschlupfdrehzahl durchgeführt. In Abb. 8 ist der Verlauf vier möglicher Ansatzfunktionen dargestellt. Basierend auf den geringen Bestimmtheitsmaßen erster linearer Regressionsmodelle (vgl. $[10,21])$ sowie an der Lage der Stützpunkte (vgl. Abb. 8a)) wird ersichtlich, dass tendenziell ein nichtlinearer Zusammenhang vorliegt. Die Ansatzfunktionen in b) (polynomiale Ansatzfunktion 2. Grades) und c) (Exponentielle Ansatzfunktion mit zwei e-Termen) liefern hohe Bestimmtheitsmaße und werden daher als mögliche Ansatzfunktionen berücksichtigt. Sowohl die hyperbelförmige Funktion in b) als auch die Funktion in c) führen im Verlauf allerdings zu einer Zunahme der Bewertungsgröße mit zunehmender Grundschlupfdrehzahl. Dies entspricht nicht dem zu erwartenden Verlauf einer reduzierten Bewertungsgröße bei zunehmender Schlupfdrehzahl aufgrund der Erhö- hung der Dämpfung. Ein exponentieller Ansatz mit einem e-Term (d)) liefert ein hohes Bestimmtheitsmaß sowie einen plausiblen Verlauf, da zu erwarten ist, dass die Bewertungsgröße mit zunehmender Schlupfdrehzahl gegen 0 konvergiert.

Es wird die exponentielle Ansatzfunktion aus Abb. 8d ausgewählt:

$f(x)=a \cdot \exp (b \cdot x)$

Die Parametrierung der Modelle wird nun exemplarisch anhand von drei Parameterkombination (drei Stützstellen) bei welchen die Grundschlupfdrehzahl variiert wird, durchgeführt. Für das spezifische Amplitudenverhältnis im Betriebsbereich ergibt sich das in Abb. 9 dargestellte Regressionsmodell mit den Parametern $a=143,4$ und $b=-0.09511$. Für die Winkelbeschleungigung wird analog vorgegangen. Der Verlauf des zugehörigen Regressionsmodell mit den Parametern $a=399$ und $b=-0.06367$ wird in Abb. 10 dargestellt.

Aus den Abbildungen wird eine hohe Übereinstimmung der Modelle mit den Messpunkten ersichtlich. Die hohen Bestimmtheitsmaße lassen auf eine gute Modellgüte rückschließen. Eine Validierung der Modelle wird in nachfolgenden Schritten durchgeführt.
Abb. 10 Angenäherter Verlauf der Winkelbeschleunigung über der Grundschlupfdrehzahl, Regressionsmodell aus 3 Stützstellen, $\mathrm{R}^{2}$ : 96,5\%; (Die Kurven mit 5 Stützstellen sind zur besseren Sichtbarkeit ausgeblendet, diese werden im Kapitel Validierung eingeblendet und mitbetrachtet.)

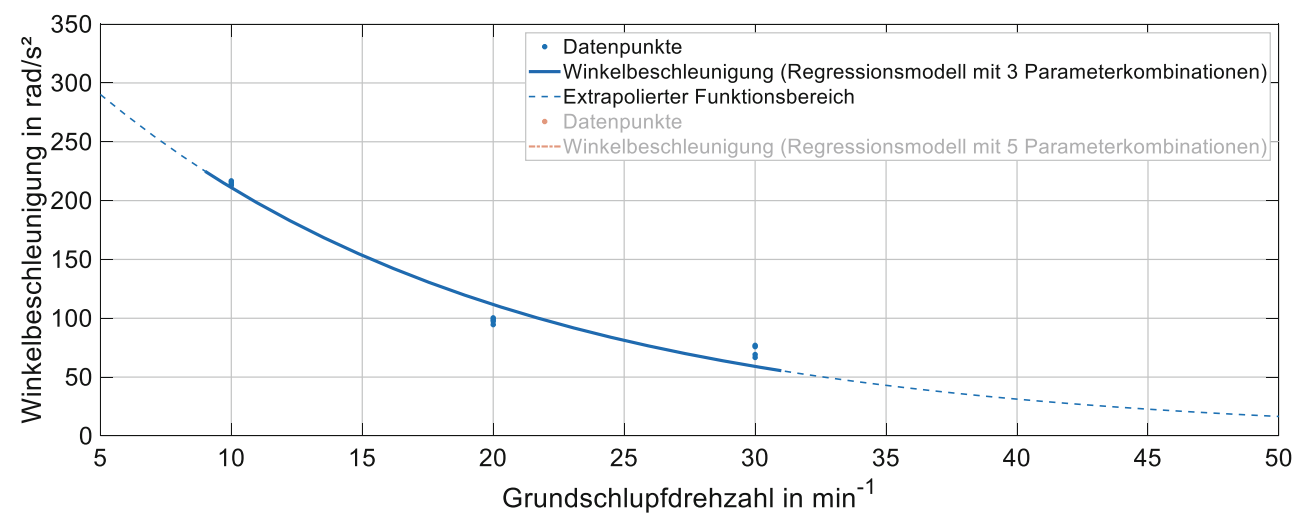


Abb. 11 Zusätzliche Messpunkte sowie Regressionsmodell basierend auf fünf Parameterkombinationen in rot zur Validierung des Regressionsmodells für den Verlauf des spezifischen Amplitudenverhältnisses

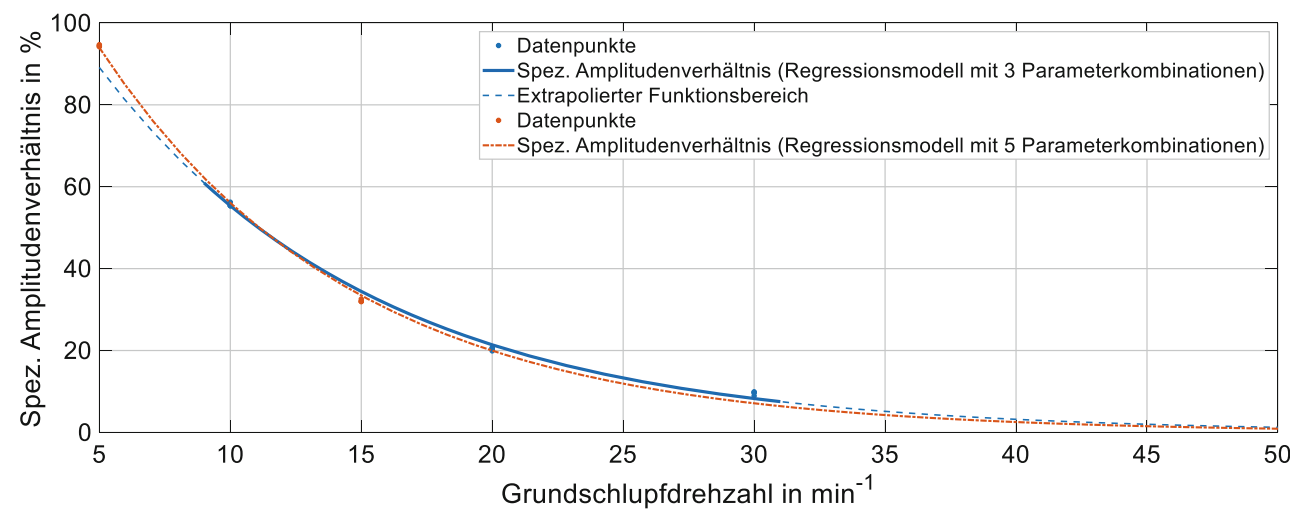

\subsection{Validierung und Modellgrenzen}

Die Validierung der Modelle wird exemplarisch anhand der zuvor ermittelten Regressionsmodelle für den Verlauf des spezifischen Amplitudenverhältnisses sowie der Winkelbeschleunigung über der Grundschlupfdrehzahl durchgeführt. Es wird sowohl eine Validierung des interpolierten als auch eine Validierung des extrapolierten Bereichs durchgeführt. Hierfür werden zwei weitere Messpunkte mit jeweils vier Einzelmessungen herangezogen. In Abb. 11 und 12 sind die jeweiligen Messpunkte mit den Grundschlupfdrehzahlen $n_{S}=5 \mathrm{~min}^{-1}$ und $n_{S}=15 \mathrm{~min}^{-1}$ in Rot dargestellt. Ergänzend wird im roten Verlauf das aus allen 5 Stützstellen ermittelte Regressionsmodell aufgetragen.

Die Abweichung der Messpunkte im interpolierten Bereich $\left(n_{S}=15 \mathrm{~min}^{-1}\right)$ liegt im Rahmen der Messschwankungsbreite. Im interpolierten Bereich kann das nichtlineare Regressionsmodell mittels der minimal notwendigen Anzahl an Stützstellen für ein nichtlineares Modell (drei) aufgestellt werden um bereits eine hinreichend genaue Aussagekraft zu gewährleisten.

Im extrapolierten Bereich geringerer Schlupfdrehzahlen kann für das Regressionsmodell der Winkelbeschleunigung eine hohe Übereinstimmung mit den gemessenen Daten identifiziert werden. Bei Schlupfdrehzahlen von $\sim n_{S}<$ $5 \mathrm{~min}^{-1}$ min kann aufgrund einer tendenziellen Veränderung von Wirkmechanismen im tribologischen Kontakt bei sehr kleinen Schlupfdrehzahlen (u.a. Neigung zu Rupfschwingungen) von keiner allgemeinen Gültigkeit des Modells ausgegangen werden. Dies lässt sich bereits an dem, gegenüber dem Modell etwas größeren experimentell ermittelten Amplitudenverhältnis für $n_{S}=5 \mathrm{~min}^{-1}$ erkennen. Der extrapolierte Bereich $n_{S}=30 \mathrm{~min}^{-1}$ wird aufgrund geringerer Relevanz (höhere Reibleistung) nicht weiter untersucht, auf Basis der Annahme von unveränderten Wirkmechanismen mit zunehmender Schlupfdrehzahl kann jedoch von einer Gültigkeit der Modelle in diesem Bereich ausgegangen werden. Im Allgemeinen kann auf Basis dieser Validierung eine Gültigkeit der aufgeführten Regressionsmodelle mit tendenziell unveränderten Wirkmechanismen in einem Bereich von Schlupfdrehzahlen $n_{S}=5$ bis $n_{S}=30 \mathrm{~min}^{-1}$ bestätigt werden.

\subsection{Skalierbarkeit der Modelle für die Veränderung weiterer Betriebsgrößen}

Die Skalierbarkeit wird für weitere identifizierte Einflussgrößen (vgl. [10]) betrachtet. Es sollen dadurch Aussagen abgeleitet werden können, wie sich die Schwingungsreduzierungswirkung bei Veränderung des Kupplungsmoments sowie bei Veränderung der eingebrachten Anregung, bspw. bei Verwendung einer 3-Zylinder anstatt einer 4-ZylinderVKM, qualitativ verhält. Für die Einflussgrößen wird der in Kap. 3 vorgestellte Untersuchungsbereich berücksichtigt.
Abb. 12 Zusätzliche Messpunkte sowie Regressionsmodell basierend auf fünf Parameterkombinationen in rot zur Validierung des Regressionsmodells für den Verlauf der Winkelbeschleunigung

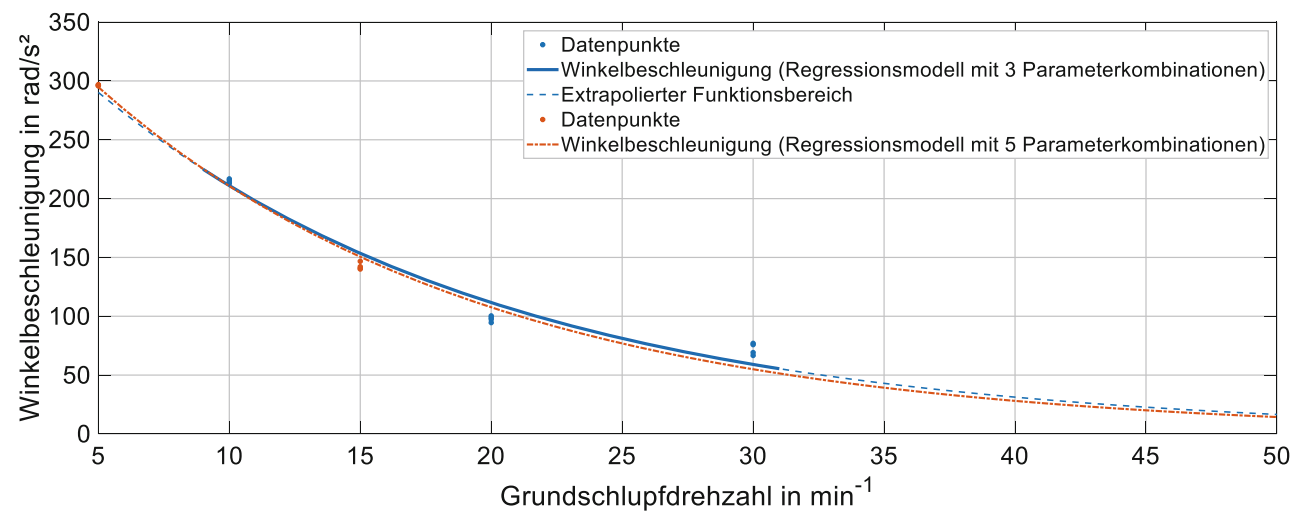


Abb. 13 Angenäherter Verlauf des spez. Amplitudenverhältnisses mit bereichsabhängigen Regressionsmodellen, $\mathrm{R}^{2}>99 \%$

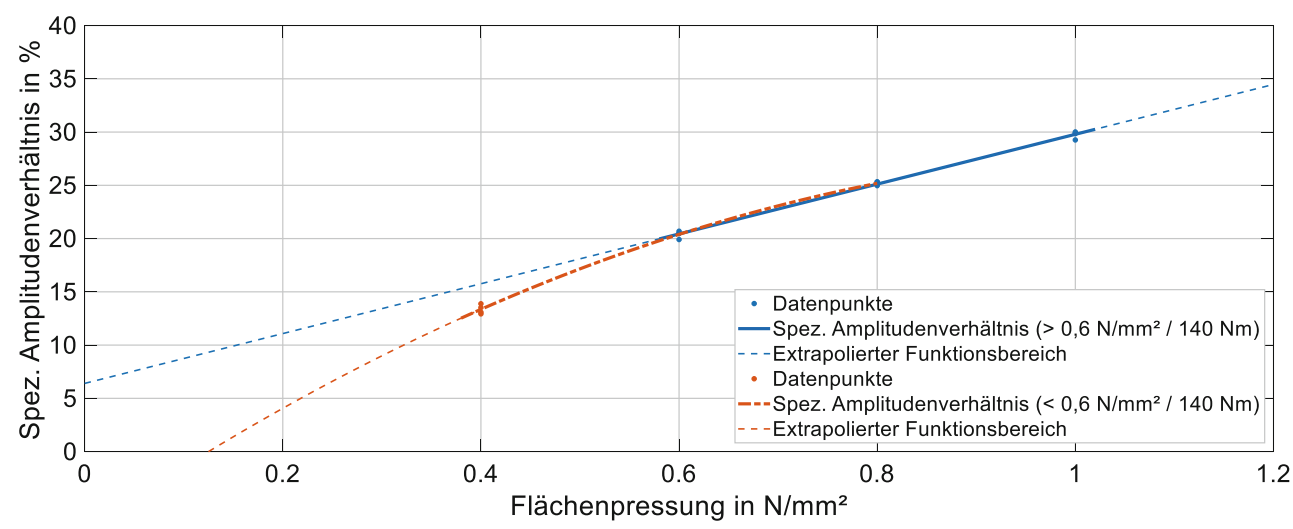

regung über das folgende Polynom 2. Grades angenähert und in einem gemeinsamen Diagramm dargestellt.

$f(x, y)=p_{00}+p_{10} \cdot x+p_{01} \cdot y+p_{20} \cdot x^{2}+p_{11} \cdot x y+p_{02} \cdot y^{2}$

Das entsprechende Kennfeld, die Bewertungsgröße spezifisches Amplitudenverhältnis nun in der farbigen z-Achse, ist in Abb. 14 dargestellt.

Aus Abb. 14 wird ersichtlich, dass ausgehend vom gewählten Betriebspunkt $\left(n_{S}=20 \mathrm{~min}^{-1}\right.$ und $\left.p=0,6 \mathrm{~N} / \mathrm{mm}^{2}\right)$ für das Modell in Abb. 9 sowohl bei einer Reduzierung der Anregungsordnung als auch bei einer Erhöhung der Anregungsamplitude eine quadratische Skalierbarkeit hin $\mathrm{zu}$ einem höheren spezifischen Amplitudenverhältnis im untersuchten Bereich mit hoher Genauigkeit abgeleitet werden kann. Zur Skalierung für z.B. eine reduzierte Anregungsordnung kann das Kennfeld in Abb. 14 herangezogen werden. Aufgrund der konstanten Antriebsdrehzahl entspricht im Kennfeld jede Anregungsordnung einer konstanten Anregungsfrequenz zwischen 20 und $60 \mathrm{~Hz}$. Die Unterschiede in der Schwingungsreduzierungswirkung der einzelnen Frequenzen können aus Wechselwirkungen mit dem Restantriebsstrangmodell resultieren. Eine andere mögliche Erklärung ist, dass die Unterschiede aus einer besseren Isolationswirkung im Friktionskontakt bei höheren Frequenzen resultiert. Diese Hypothesen gilt es in weiteren Untersuchungen zu veri- oder falsifizieren.

\subsection{Weitere Kennfelder \& Schwingungsreduzierungswirkung - Reibleistung}

Ergänzend werden auf Basis der Messdaten zwei anwendungsorientierte Kennfelder ermittelt. Möchte der Anwender nun ableiten, welche Schlupfdrehzahl bei entsprechender Flächenpressung eingestellt werden muss, um eine gewünschte Schwingungsreduzierung zu erzielen, kann dazu ein solches Kennfeld herangezogen werden. Abb. 15 zeigt ein, exemplarisch für das untersuchte tribologische System erarbeitetes Kennfeld für das Verhalten der Schwin- 


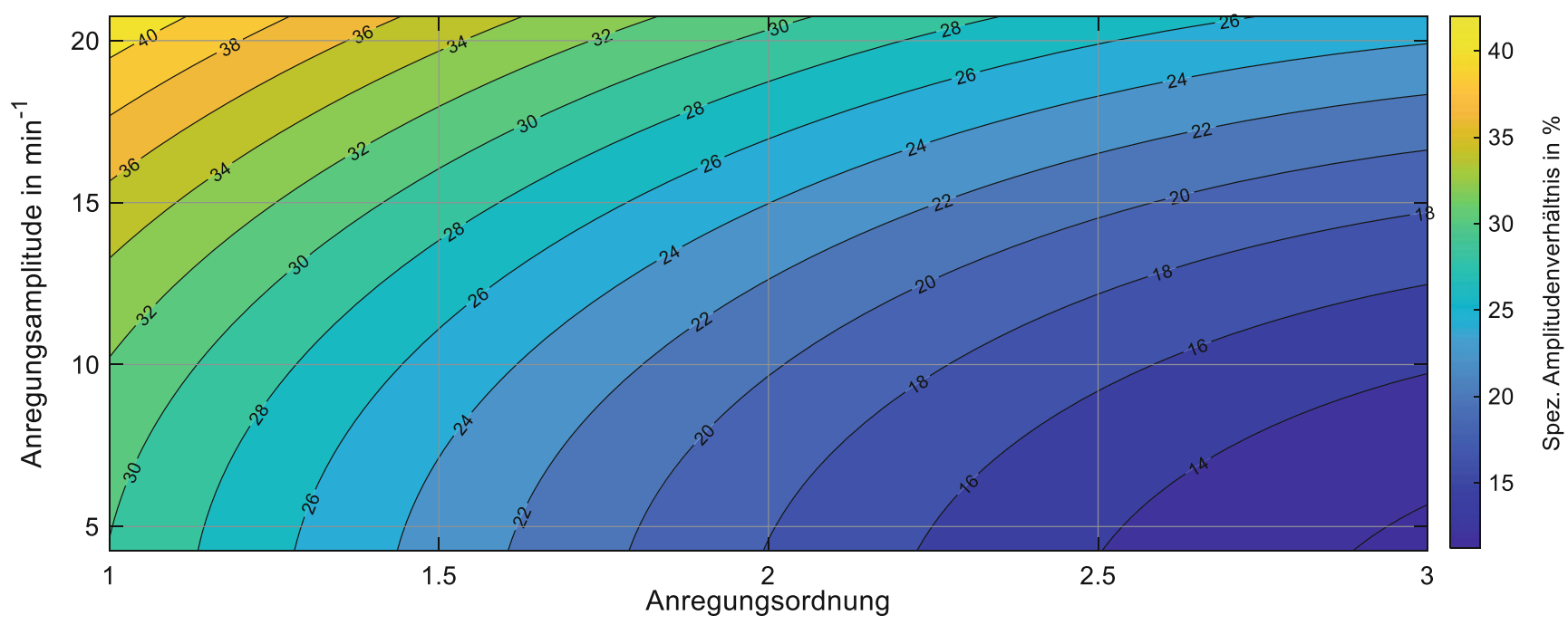

Abb. 14 Kennfeld für das spezifische Amplitudenverhältnis über Anregungsordnung und Anregungsamplitude, $\mathrm{R}^{2}=95,5 \%$

gungsreduzierung bei Veränderung von Grundschlupfdrehzahl und/oder Flächenpressung. Dabei wird entsprechend der Erkenntnisse aus vorangehender Modellbildung und Skalierbarkeit im Kennfeldbereich für den Einfluss der Flächenpressung ein linearer Ansatz, für den Einfluss der Schlupfdrehzahl ein quadratischer Ansatz verwendet. Das jeweilige Kupplungsmoment korreliert sowohl mit der Flächenpressung als auch mit der Schlupfdrehzahl, da die Veränderung dieser mit einer Veränderung der Reibungszahl im Friktionskontakt einhergeht. Im untersuchten tribologischen System und abgebildeten Untersuchungsbereich reicht das Kupplungsmoment von $120 \mathrm{Nm}$ für $n_{S}=5 \mathrm{~min}^{-1}$ und $p=0,6 \mathrm{~N} / \mathrm{mm}^{2}$ bis $210 \mathrm{Nm}$ für $n_{S}=25 \mathrm{~min}^{-1}$ und $p=1,0 \mathrm{~N} / \mathrm{mm}^{2}$. Zur Orientierung kann das Kupplungsmoment bei $n_{S}=20 \mathrm{~min}^{-1}$ betrachtet werden, welches hier mit Erhöhung der Flächenpressung entlang der grauen Linie von 140 auf $205 \mathrm{Nm}$ ansteigt. Untersuchungen zum kupplungsmomentunabhängigen Einfluss der Flächenpressung sind hingegen Teil zukünftiger Untersuchungen und können z.B. durch eine Variation der Lamellenanzahl und damit Veränderung der Anzahl an Reibflächen durchgeführt werden.

Für den Zusammenhang zwischen Schwingungsreduzierung und Reibleistung im untersuchten tribologischen System wird das in Abb. 16 dargestellte Kennfeld für drei unterschiedliche Flächenpressungen bzw. Kupplungsmomentbereiche erarbeitet. Das Kennfeld kann für quantative Aussagen über die zu erwartende Verlustleistung bei einem gewünschtem Reduzierungsgrad herangezogen werden.

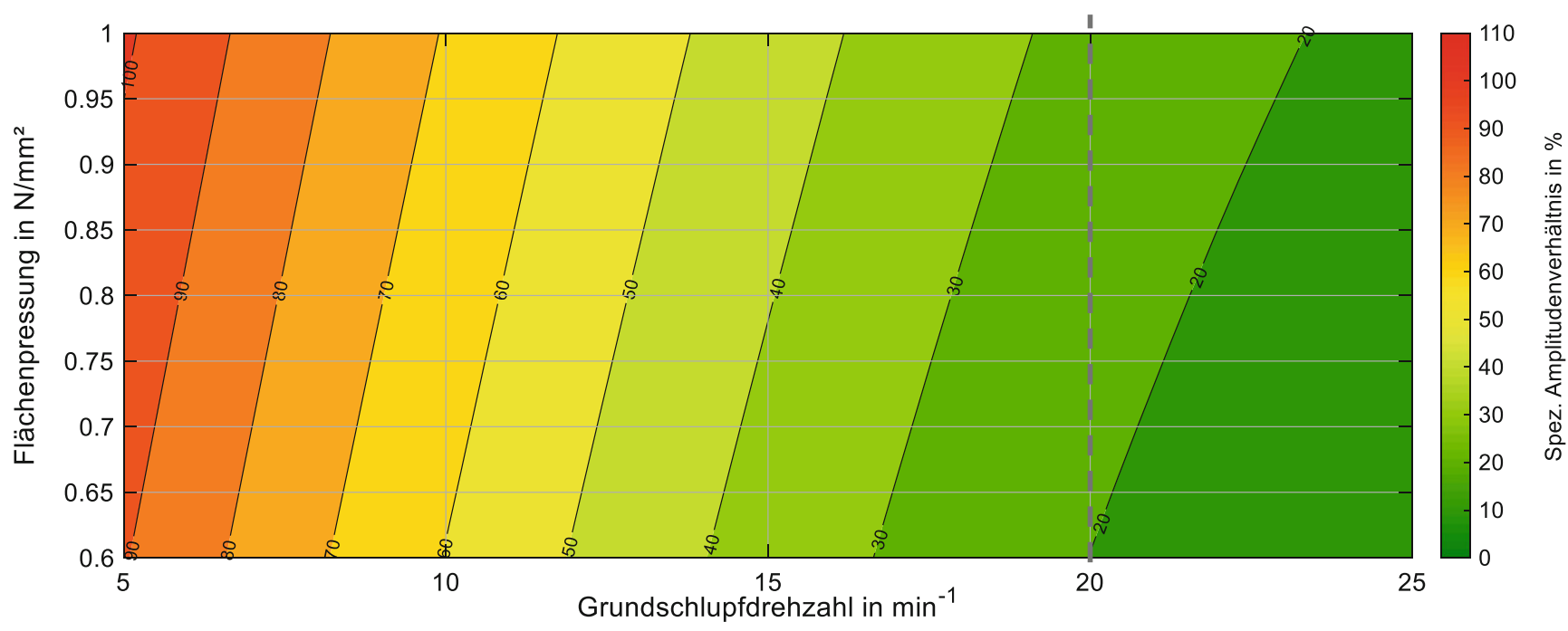

Abb. 15 Kennfeld für das spezifische Amplitudenverhältnis über Grundschlupfdrehzahl und Flächenpressung für Kupplungsmomente im Bereich von 120 bis $210 \mathrm{Nm}$ (abh. von $p$ und $n s), \mathrm{R}^{2}: 99,5 \%$ 
Abb. 16 Interpolierter Verlauf des spez. Amplitudenverhältnisses über der spez. Verlustleistung für drei Flächenpressungen, $\mathrm{R}^{2}: 99,7$ bis $99,9 \%$

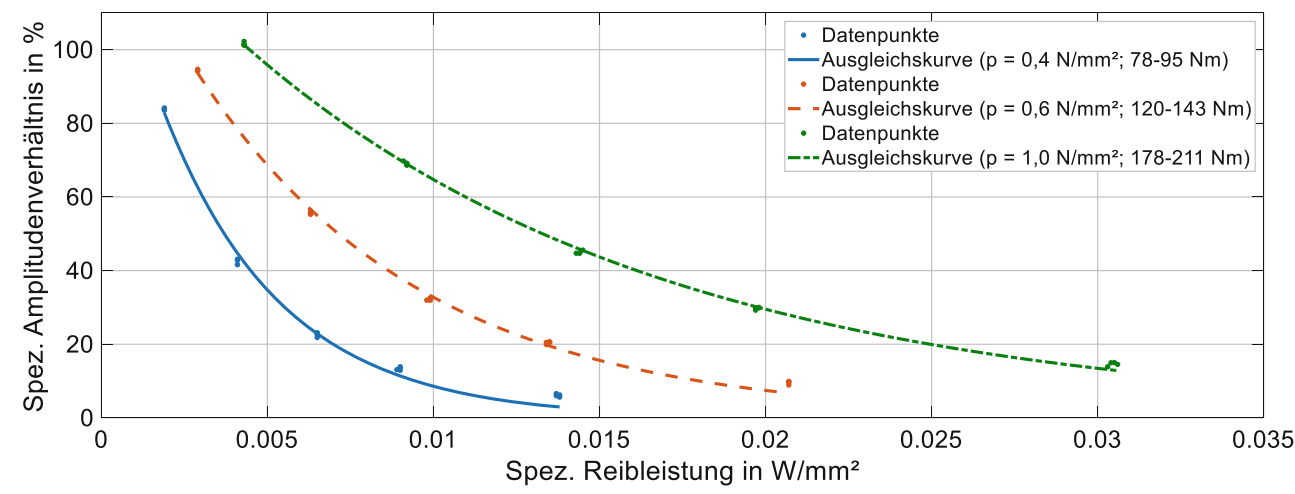

Je geringer die Flächenpressung bzw. das Kupplungsmoment, desto günstiger ist der Verlauf der Zielkonfliktkurve für Schwingungsreduzierung und Verlustleistung. Im untersuchten Kupplungssystem liegen vier Reibpaarungen (Wirkflächenpaare) vor. Eine Hochrechnung auf ein reales Kupplungssystem mit mehr Lamellen bzw. Reibpaarungen ist nicht direkt möglich, da mit mehr Wirkflächenpaaren auch mehr Energie durch Dämpfung dissipiert werden kann, was die Schwingungsreduzierung verbessern könnte und die Kurven in Abb. 16 entsprechend nach links unten verschieben würde. Allgemein lässt sich hier aber festhalten, dass im untersuchten System bei Kupplungsmomenten im Bereich $\sim 180-210 \mathrm{Nm}$ eine Reibleistung von in etwa $0,5 \mathrm{~kW}$ notwendig ist, welche hier einer anteiligen Verlustleistung der eingebrachten Leistung von ca. $2 \%$ entspricht, um die aufgebrachte Schwingungsamplitude um $80 \%$ (entspricht Amplitudenverhältnis Ausgang/Eingang von 20\%) zu reduzieren.

\section{Zusammenfassung und Ausblick}

In dieser Veröffentlichung wird der Einfluss von Betriebsgrößen auf die Schwingungsreduzierungswirkung mittels empirischer Modellbildung qualitativ und für ein exemplarisches Tribosystem quantitativ ermittelt. Es werden dazu empirisch nichtlineare Modelle für das Verhalten der Bewertungsgrößen Amplitudenverhältnis und Winkelbeschleunigung bei Veränderung folgender Betriebsgrößen erarbeitet:

- Grundschlupfdrehzahl,

- Flächenpressung bzw. Kupplungsmoment,

- Amplitude und Ordnung der Anregung.

Zwei relevante Parameterräume werden auf Basis vorangehender Untersuchungen abgeleitet. In der vorgestellten Validierungsumgebung zur dynamischen Untersuchung des Übertragungsverhaltens in nasslaufenden Lamellenpaketen wird experimentell die Schwingungsreduzierung in festgelegten Betriebspunkten ermittelt. Auf Basis der er- mittelten Stützstellen werden Regressionsmodelle errechnet. Die Modelle werden durch Messdaten validiert und auf deren Modellgrenzen sowie deren Gültigkeit hin untersucht. Ergänzend wird die Skalierbarkeit, bspw. bei Veränderung der Hauptanregenden, anhand eines Modells exemplarisch untersucht. Es wird gezeigt, dass das Schwingungsreduzierungsverhalten des nasslaufenden Lamellenpakets im Schlupfbetrieb bei Veränderung der Betriebsgrößen innerhalb der ermittelten Modellgrenzen sowie in Teilen auch in extrapolierten Bereichen mittels nichtlinearer Modelle hinreichend genau (Bestimmtheitsmaße > 95\%) abgebildet werden kann. Es werden Modelle mit hoher Genauigkeit erarbeitet.

Um in zukünftigen Auslegungen eine Abschätzung des Zielkonflikts Komfort vs. Effizienz im schlupfbetriebenen Kupplungssystem begünstigen zu können, wird ein anwendungsorientiertes Kennfeld für die zu erwartende Reibleistung bei entsprechender Amplitudenreduzierung im untersuchten System ermittelt. Dabei wird eine quantifizierte Aussage über die zu erwartenden Verlustleistungen getroffen. Durch die Modelle wird die Entwicklung und Auslegung von zukünftigen Kupplungssystemen sowie Antriebssträngen in Hinblick auf eine bedarfsgerechte, gezielte Einstellung von Betriebsgrößen im dynamischen Dauerschlupfbetrieb begünstigt. Eine Komfortabschätzung und Strategieableitung hinsichtlich schwingungsmindernder Komponenten im frühen Produktentstehungsprozess wird unterstïtzt.

$\mathrm{Da}$ auch das tribologische System selbst einen signifikanten Einfluss auf die Schwingungsreduzierungswirkung im Friktionskontakt hat [22], können die Modelle in erster Linie für qualitative Tendenzen verwendet werden. Um Aussagen zur Skalierbarkeit bei einer Veränderung des tribologischen Systems, z. B. Veränderung der Ölvariante oder der Reiblamellen, treffen zu können, gilt es zukünftig die Zusammenhänge zwischen Tribosystem und Schwingungsreduzierung zu erarbeiten. Dabei gilt es auch die Kenntnisse über Wirkmechanismen im schlupfenden Friktionskontakt zu erweitern, um zukünftig auch erweiterte physikalische Zusammenhänge herstellen zu können. 
Danksagung Die durchgeführten Untersuchungen basieren mitunter auf Erkenntnissen aus dem IGF-Vorhaben 18501-N. Die Autoren danken für die Unterstützung des Forschungsprojekts. Das IGF-Vorhaben 18501-N der Forschungsvereinigung Antriebstechnik e. V. (FVA) wurde über die AiF im Rahmen des Programms zur Förderung der Industriellen Gemeinschaftsforschung (IGF) vom Bundesministerium für Wirtschaft und Energie aufgrund eines Beschlusses des Deutschen Bundestages gefördert.

Funding Open Access funding enabled and organized by Projekt DEAL.

Interessenkonflikt A. Bischofberger, S. Ott und A. Albers geben an, dass kein Interessenkonflikt besteht.

Open Access Dieser Artikel wird unter der Creative Commons Namensnennung 4.0 International Lizenz veröffentlicht, welche die Nutzung, Vervielfältigung, Bearbeitung, Verbreitung und Wiedergabe in jeglichem Medium und Format erlaubt, sofern Sie den/die ursprünglichen Autor(en) und die Quelle ordnungsgemäß nennen, einen Link zur Creative Commons Lizenz beifügen und angeben, ob Änderungen vorgenommen wurden.

Die in diesem Artikel enthaltenen Bilder und sonstiges Drittmaterial unterliegen ebenfalls der genannten Creative Commons Lizenz, sofern sich aus der Abbildungslegende nichts anderes ergibt. Sofern das betreffende Material nicht unter der genannten Creative Commons Lizenz steht und die betreffende Handlung nicht nach gesetzlichen Vorschriften erlaubt ist, ist für die oben aufgeführten Weiterverwendungen des Materials die Einwilligung des jeweiligen Rechteinhabers einzuholen.

Weitere Details zur Lizenz entnehmen Sie bitte der Lizenzinformation auf http://creativecommons.org/licenses/by/4.0/deed.de.

\section{Literatur}

1. Schaeffler Technologies AG \& Co. KG (Hrsg) (2018) obility for tomorrow-Schaeffler Kolloquium 2018. Schaeffler Technologies, Herzogenaurach

2. Albers A (1990) Elektronisches Kupplungsmanagement (EKM) Die mitdenkende Kupplung. In: 4. Internationales Kolloquium Torsionsschwingungen im Antriebsstrang, 20. April 1990, Baden-Baden

3. Lutz D, Verein Deutscher Ingenieure (1988) Kupplungsmanagement - ein Baustein zur Drehschwingungsdämpfung. VDI Berichte, Bd. 697, S 219-256

4. Jürgens G, Fischer R (1988) Vergleich verschiedener Systeme zur Verringerung von Triebstrangschwingungen, S 233-256

5. Drexl H-J, Verein Deutscher Ingenieure (1988) Der Torsionsdämpfer in der Kupplungsscheibe. VDI Berichte, Bd. 697, S 133-158

6. Abbassi MB (1999) Steigerung des Antriebsstrangkomforts im Kfz durch elektronisches Kupplungsmanagement. ATZ 101(2):118-126

7. Reik W, Friedmann O, Agner I, Werner O (2004) Die Kupplung das Herz des Doppelkupplungsgetriebes. In: Getriebe in Fahrzeugen 2004. Tagung Friedrichshafen, 22. und 23. Juni 2004. VDIBerichte, Bd. 1827. VDI, Düsseldorf, S 65-88
8. Wienholt HW, Zdora D (1995) Auslegung der Überbrückungskupplung mit geregeltem Schlupf bei Einsatz des Drehmomentwandlers in Pkw-Automatikgetrieben, S 281-299

9. Zhou B, Lu T, Zhang J, Hao H (2017) Micro-slip control and torque estimation for dual clutch transmission. Proc Inst Mech Eng Part K J Multibody Dyn 231(4):750-764

10. Bischofberger A, Ott S, Albers A (2020) Einfluss von Beanspruchungsgrößen auf die Schwingungsentkopplung im nasslaufenden Kupplungssystem und deren Korrelation mit dem Reibverhalten im Friktionskontakt. Tribol Schmierungstech 67(1):7-15

11. Kemper C, Basiewicz M, Ott S, Albers A (2018) Schwingungsentkopplung durch nasslaufende Funktionsreibkontakte im geregelten Schlupfbetrieb - Anforderungen an den tribologischen Kontakt in Wechselwirkung mit der Antriebssystemdynamik. In: 59. Tribologie-Fachtagung 2018. Göttingen

12. Kemper C, Wantzen K, Bischofberger A, Ott S, Albers A (2019) Vergleich von Reibungszahlverläufen von nasslaufenden Friktionskontakten im gezielten Schlupfbetrieb unter dynamischer Anregung. Forsch Ingenieurwes 83(2):239-246

13. Höhn B-R, Pflaum H, Hämmerl B (1997) Ermittlung der Leistungsgrenze ölgekühlter Lamellenkupplungen anhand von Temperaturkriterien. VDI Berichte, Bd. 1323, S 507-525

14. Albers A, Bischofberger A, Ott S (2020) Wet clutch as an enabler of cost-efficient hybrid drive systems decoupling as functional extension. In: 20. Internationales Stuttgarter Symposium. Automobilund Motorentechnik. Springer Vieweg, Wiesbaden, S 251-265

15. Albers A, Ott S, Basiewicz M (2017) Influencing factors on the decoupling of induced rotational oscillation by wet-running multiplate-packages in controlled slip mode. In: 44. Leeds-Lyon Symposium on Tribology. Lyon

16. Ott S, Basiewicz M (2017) Schwingungsreduzierung durch das Kupplungssystem im gezielten Schlupfbetrieb. Kupplungen und Kupplungssysteme in Antrieben. VDI, Ettlingen, Düsseldorf

17. Ott S, Basiewicz M (2017) Innovative validation environments and methods for holistic clutch system development. In: Antriebstechnisches Kolloquium ATK 2017. Tagungsband, Bd. 17, S 12

18. Albers A, Ott S, Basiewicz M (2017) Ermittlung des Übertragungs- und Systemverhaltens nasslaufender Lamellenpakete im Schlupfbetrieb unter Berücksichtigung der Systemwechselwirkungen. Kupplungen und Kupplungssysteme in Antrieben. VDIVerlag, Ettlingen, Düsseldorf

19. Czichos H, Habig K-H (Hrsg) (2015) Tribologie-Handbuch. Tribometrie Tribomaterialien Tribotechnik. SpringerLink Bücher. Springer Vieweg, Wiesbaden

20. GfT Gesellschaft für Tribologie e. V. (Hrsg) (2018) 59. TribologieFachtagung 2018. Göttingen

21. Bischofberger A, Ott S, Albers A (2020) Schwingungsreduzierung Nasslauf. Einflussgrößen auf die Entkopplung von induzierten Drehungleichförmigkeiten durch nasslaufende Kupplungssysteme im Schlupfbetrieb. Abschlussbericht FVA-Nr. 780 I. FVA-Heft, Bd. 1388. FVA Forschungsvereinigung Antriebstechnik e. V., Frankfurt a.M.

22. Bischofberger A, Ott S, Albers A (2020) Die nasslaufende Kupplung als Stellglied zur Schwingungsreduzierung im Antriebsstrang - Einflüsse eines veränderten Tribosystems. Forsch Ingenieurwes $85: 1-10$ 